have been present in the histidine and tryptophane preparations used could not account for their entire lack of activating influence upon the amyloclastic action of the enzyme.

We are greatly indebted to the Carnegie Institution of Washington for grants in aid of this investigation.

NEW YORK, N. Y.

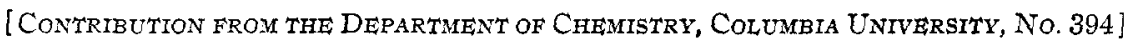

\title{
INFLUENCE OF LYSINE UPON THE HYDROLYSIS OF STARCH BY PURIFIED PANCREATIC AMYLASE
}

By H. C. Sherman and Mary L. Caldweli

Received August 3, 1922

The studies of the influence of certain natural amino acids on the hydrolysis of starch by pancreatic amylase already reported from this Laboratory ${ }^{1}$ have been extended to lysine with results which throw interesting light upon the nature of the enzyme.

The experimental methods were essentially the same as those used in the work with other amino acids and the precautions regarding the selection and care of glass ware, the purification of the water and other materials, the exclusion of light, and the standard conditions of temperature, activating salts, ${ }^{2}$ and hydrogen-ion concentration ${ }^{3}$ repeatedly emphasized in reports from this Laboratory were carefully observed.

The lysine hydrochloride used was prepared from lysine picrate by the method described by Kossel. ${ }^{4}$ The picrate was dissolved in an aqueous solution of hydrochloric acid and the picric acid removed by extracting with ether. This was repeated several times after the ether extract upon evaporation gave no color, taste, or weighable residue. The lysine hydrochloride was obtained by evaporating the solution with excess of hydrochloric acid and recrystallizing several times from absolute alcohol. The product obtained was white, crystalline, hygroscopic, and had the melting point of the dihydrochloride $\left(192-193^{\circ}\right)$. Purified pancreatic amylase No. $24 \mathrm{~B}$ prepared as previously described ${ }^{6}$ was used.

Lysine was tested for its influence upon both the amyloclastic and the saccharogenic activities of the amylase by the methods previously described. ${ }^{1}$ The calculated weight of lysine dihydrochloride needed to give the desired amount of lysine was added directly to the fully activated starch paste in the cylinder before making it up to volume and enough

${ }^{1}$ Sherman and Walker, THrs JournaL, 41, 1866 (1919); 43, 2461 (1921). Sherman and Caldwell, ibid., 43, 2469 (1921).

2 Sherman and Kendall, ibid., 32, 1073 (1910), and subsequent papers from this Laboratory.

${ }^{3}$ Sherman, Thomas and Baldwin, ibid., 41, 231 (1919).

4 Kossel, Z. physiol. Chem., 26, 586 (1898-9).

s Abderhalden's "Biochemisches Handlexikon," Julius Springer, vol. IV, 645 (1911).

- Sherman and Neun, This Journal, 41, 1854 (1919). 
$0.01 \mathrm{~N}$ sodium hydroxide solution was added to bring the hydrogen-ion concentration in the starch paste to the optimum, ${ }^{3} C_{\mathrm{H}^{+}}=10^{-6.93}$.

The expression "fully activated" here means that the starch dispersion contained optimum concentrations of chloride and phosphate. The hydrogen-ion concentrations were determined in an aliquot part of each portion of starch paste prepared. This was done by both the electrometric and the colorimetric methods. In the latter, Sörensen's phosphate buffer mixtures, ${ }^{7}$ standardized electrometrically, were used.

Since glycine had been found by previous work ${ }^{1}$ to have a favorable influence on both the amyloclastic and saccharogenic activities of pancreatic amylase, it was decided to test the influence of lysine side by side with that of equal weights of glycine. The results of a typical test on the amyloclastic activity of this amylase are given in Table $I$.

TABLE I

The Influence of Lysine as Compared with Glycine upon the Amyloclastic Activity of Purified Pancreatic Amylase

\begin{tabular}{|c|c|c|c|c|c|c|c|}
\hline \multicolumn{2}{|c|}{$\begin{array}{l}\text { Substance } \\
\text { added }\end{array}$} & \multirow[t]{2}{*}{$\stackrel{\log }{\mathrm{C}_{\mathrm{B}}+}$} & & \multicolumn{2}{|c|}{ Enzyme in mg. } & \multicolumn{2}{|c|}{$\begin{array}{l}\text { "Amyloclastic } \\
\text { power" } \times 10^{-3}\end{array}$} \\
\hline $\mathrm{Mg}$. & Amino Acid & & 0.006 & 0.0065 & 0.007 & 0.0075 & \\
\hline & control & -6.93 & V.R. & V.R. & V.R. & $\mathrm{R}^{a}$ & 666 \\
\hline 50 & lysine " $A$ " & -6.93 & V.R. & V.R. & V.R. & $\mathrm{R}^{a}$ & 666 \\
\hline 50 & lysine "C" & -6.93 & V.R. & V.R. & V.R. & $\mathrm{R}^{a}$ & 666 \\
\hline 50 & glycine & -6.93 & V.R. & $\mathrm{R}^{a}$ & O.R. & R.O. & 769 \\
\hline & control & -6.93 & V.R. & V.R. & V.R. & $\mathrm{R}^{a}$ & 666 \\
\hline
\end{tabular}

17 The end-point tube is that one of lowest enzyme concentration which is definitely red and shows no blue or violet color due to starch. The terms used in describing the colors are those of the Milton Bradley Standard Color Chart as given by Mulliken in his "Identification of Pure Organic Compounds," John Wiley and Sons, New York, 1904.

The amyloclastic power of the enzyme is obtained by dividing the weight of $1 \%$ starch paste $(5000 \mathrm{mg}$.) by the weight in milligrams of enzyme present in the tube showing the end-point.

The "controls" are blank determinations made up of starch pastes neutralized and containing the proper concentrations of inorganic salts but no amino acids. These are used as the standard each time, as results vary slightly from day to day.

The results given above again show that glycine has a definitely favorable influence on the amyloclastic activity of the amylase, while lysine has no apparent effect. Similar results had previously been obtained with histidine and tryptophane. ${ }^{1}$

A study was next made of the influence of lysine as compared with glycine upon the saccharogenic activity of the amylase. Typical results are given in Table II, which show that unlike the tests upon the amylo-

"Clark, "The Determination of Hydrogen Ions," Williams and Wilkins Co., 1920, p. 81. 
clastic activity, a consistently favorable action is obtained by lysine as well as glycine when tested in equal amounts, under standard conditions, for its effect on the saccharogenic activity of this amylase. The results are given in terms of cuprous oxide formed by the reduction of Fehling solution by the sugar or sugars produced by the digestion of the starch by the amylase.

\section{TABLE II}

Influence of Lysine and Glycine on the Saccharogenic Activity of Purified PANCREATIC AMYlase

Additions to digestion mixture

Mg. Amino acid

None

50 glycine

50 lysine "A"

50 lysine "B"
Cuprous oxide

obtained

$\mathrm{Mg}$.

$207 \quad$. $\quad-6.93$

$266-6.93$

$242-6.93$

$248-6.93$

\section{Discussion}

The contrasting results thus obtained from lysine according as its effect is tested upon the amyloclastic or the saccharogenic action of the pancreatic amylase are of special interest, since in previous studies these two phases of the activity of this enzyme had been found to run practically parallel. ${ }^{8}$ The quantitative values of the amyloclastic and saccharogenic powers of different preparations from the pancreas show an approximately constant relation to each other. ${ }^{8}$ The same concentrations of hydrogen ion and of sodium chloride and disodium phosphate appear to be required for optimum amyloclastic as for optimum saccharogenic activity. When 2 representative mono-amino acids, glycine and phenylalanine, were tested for their influence first on the saccharogenic ${ }^{1}$ and then on the amyloclastic ${ }^{1}$ activity of pancreatic amylase, both amino acids showed favorable effects in each case. It appears probable that the difference in the case of lysine, in that it shows no effect when tested upon amyloclastic, but does show a favorable influence in tests upon the saccharogenic action, is not due simply to differing sensitivity of method, but rather to the fact that amyloclastic and saccharogenic action are to some extent different properties of the amylase. In the absence of other evidence these two activities would usually be attributed to the presence of 2 different enzymes in the amylase preparations used. But the ratio of amyloclastic to saccharogenic activities of the purified amylase preparations remains practically the same as in the original pancreatin from which they are made, and it appears improbable that two different enzymes could pass through the various operations of the purification process and remain in the same quantitative relation to each other.

Of particular interest is the fact that it is the first (amyloclastic) phase

sherman and Schlesinger, This Journal, 35, 1784 (1913). 
of the enzyme's activity which is not influenced by lysine, while the later (saccharogenic) phase is favorably affected.

The purified preparations of this enzyme (pancreatic amylase) as obtained in this Laboratory have been shown to be essentially protein ${ }^{9}$ and much experimental evidence has also been given which would lead to the conclusion that the amylase itself is of a protein nature. The work on the influence of amino acids previously published from this Laboratory emphasizes this view. If the enzyme is protein and its deterioration in the water solutions in which it acts is due to hydrolysis, the addition of amino acids would tend to conserve the active molecule by retarding its hydrolysis and thus would have an apparent "activating" effect. It has been shown experimentally that addition of certain amino acids does certainly lessen the deterioration of the enzyme which is so striking a characteristic of purified pancreatic amylase in aqueous solution.

That lysine fails to influence amyloclastic activity is an indication that it is so bound in the enzyme molecule as not to be liberated by hydrolysis until after the enzyme has passed the amyloclastic stage of its action, which is a property having to do with the earliest stage of the starch hydrolysis; whereas the saccharogenic activity is (for the most part at least) exerted later, and in this later phase of its activity the enzyme (which moy have already lost its amyloclastic power) is to a measurable extent protected by the presence of lysine from such further hydrolytic change as would result in the loss of its saccharogenic activity.

\section{Summary}

Lysine shows no effect upon the amyloclastic action of pancreatic amylase but exerts a favorable influence upon its saccharogenic action.

These facts are discussed in the light of our previous observations upon this and other amylases.

According to the view that the enzyme (pancreatic amylase) is essentially a protein substance which gradually becomes inactivated through hydrolysis in the aqueous medium in which it acts, and that the apparent "activating" influence of amino acids is due to retardation of this hydrolysis of the enzyme, it appears probable that the 1ysine in the enzyme molecule is so bound that it would not in any case be split off until after the stage of amyloclastic action had been passed, but that it (lysine) is concerned in the later stages represented by saccharogenic activity and, therefore, the addition of lysine to the enzyme solution tends to conserve the enzyme by diminishing the rate of its further hydrolysis at this later stage.

The observations upon lysine thus confirm and extend the theory which we advanced last year in explanation of our finding that histidine and tryp-

'Sherman and Schlesinger, ThIs Journal, 37, 1305 (1915). Sherman and Gettler, ibia.. 35, 1790 (1913). Ref. 6. 
tophane do not favor the amyloclastic activity of this enzyme as do the mono-amino acids tested.

We are greatly indebted to the Carnegie Institution of Washington for grants in aid of this investigation.

NEW YORE, N. Y.

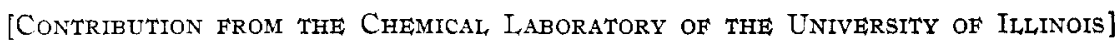

\section{OPTICALLY ACTIVE DYES. I}

By A. W. INGERSOLL, WITH ROGER ADAMS ${ }^{1}$

Received August 14, 1922

The nature of the action by which dyes are absorbed and more or less permanently held by animal or vegetable fibers, has been the subject of a large number of investigations. The first work recorded was during the eighteenth century and since then the experiments in this field have been very extensive. ${ }^{2}$ From results so far obtained, no definite conclusions can be drawn as to whether there is involved a physical or chemical phenomenon or both. Scores of articles have been published by many authors, some of whom are advocates of a physical, some of a chemical explanation.

An excellent method which should throw light upon this subject is the study of the action of optically active dyes upon fibers. Each of a pair of mirror images would be expected to have the same dyeing properties, if the absorption by the fibers is purely a physical process. On the other hand, if a chemical reaction of any sort is taking place when the dye is absorbed, a different degree or rate of absorption of the $d$ and $l$ forms might take place on account of the optical activity of the substances of which the fibers are composed. In this connection, however, it must be considered that even though the dyeing process may be a chemical one, the $d$ and $l$ forms of every pair of dyes would not necessarily be absorbed to a different degree, sufficient to be measured. Only after the study of a large number of such pairs could a convincing conclusion be drawn.

Very little work upon the selective absorption of one active compound as compared with its enantiomorph has been carried out and no pairs of optically-active dyes have been made. In 1904, Willstaetter ${ }^{3}$ discussed the possibility of selective absorption by wool of one active component of a racemic mixture. He carried out experiments upon solutions of

1 This communication is an abstract of a thesis submitted by A. W. Ingersoll in partial fulfilment of the requirements for the degree of Doctor of Philosophy in Chemistry at the University of Illinois.

${ }^{2}$ An excellent review of the articles on the theory of dyeing from the earliest times may be found in the following books: Georgievics, "Chemistry of Dyestuffs," Scott, Greenwood and Co., 1903; Dreaper, "Chemistry and Physics of Dyeing," P. Blakiston's Son and Co., 1906; Knecht, Rawson and Loewenthal, "A Manual of Dyeing," Charles Griffin and Co., 1910; Wood, "Chemistry of Dyeing," D. Van Nostrand Co., 1913.

${ }^{3}$ Willstaetter, Ber., 37, 3758 (1904). 